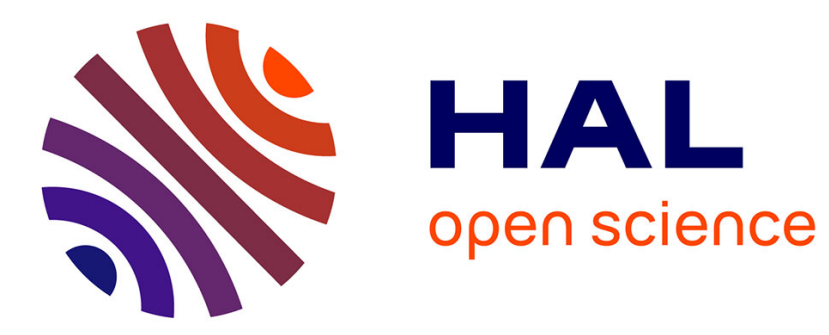

\title{
Muslime im heutigen Spanien
}

Claude Proeschel

\section{To cite this version:}

Claude Proeschel. Muslime im heutigen Spanien. Aslan, Edgan (Hg)), Islamic Education in Europa, Böhlau Verlag, pp.15-30, 2009. halshs-00278383

\section{HAL Id: halshs-00278383 https://shs.hal.science/halshs-00278383}

Submitted on 13 May 2008

HAL is a multi-disciplinary open access archive for the deposit and dissemination of scientific research documents, whether they are published or not. The documents may come from teaching and research institutions in France or abroad, or from public or private research centers.
L'archive ouverte pluridisciplinaire HAL, est destinée au dépôt et à la diffusion de documents scientifiques de niveau recherche, publiés ou non, émanant des établissements d'enseignement et de recherche français ou étrangers, des laboratoires publics ou privés. 


\section{LES MUSULMANS DANS L'ESPAGNE D'AUJOURD'HUI}

\section{CLAUDE PROESCHEL}

Groupe Sociétés, Religions, Laïcités, GRSL(EPHE-CNRS), Paris

\section{En guise d'introduction : l'influence arabe et musulmane sur la société espagnole : deux visions}

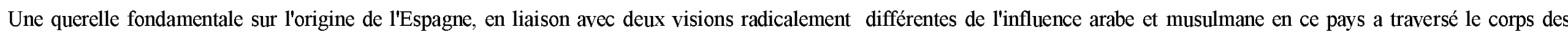

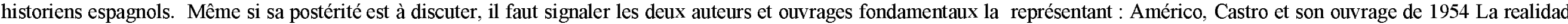

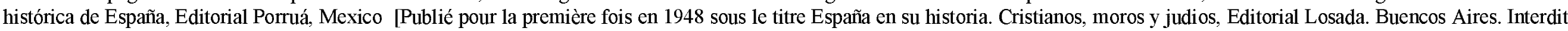

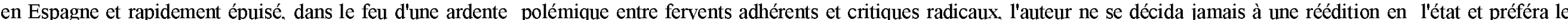

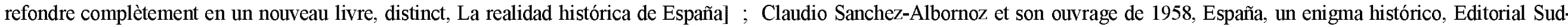

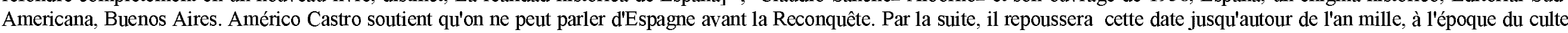

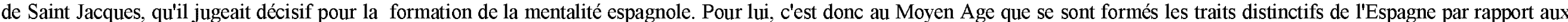

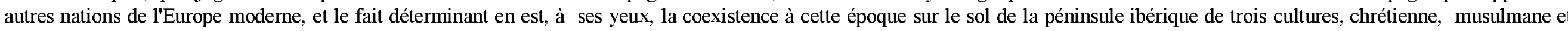

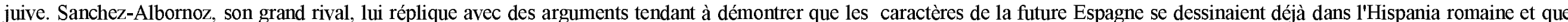

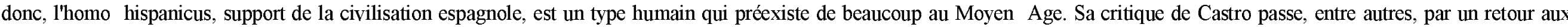

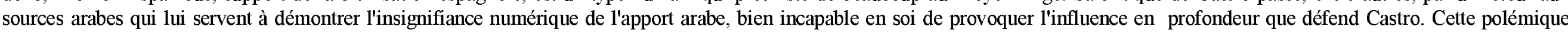

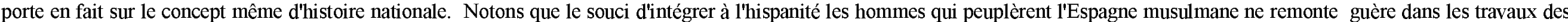
historiens au delà du XIXe siècle. On distingue plus volontiers les maures des chrétiens comme appartenant à deux entités historiques différentes.

\section{Musulmans d'Espagne}

\subsection{Présence musulmane en Espagne : contextualisation historique}

L'Espagne a construit son unité politique et culturelle par référence exclusive au catholicisme, dans la forme la plus radicale possible, par l'expulsion et la persécution des autres

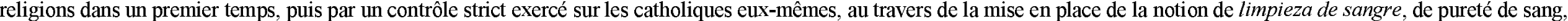

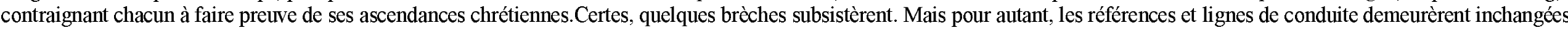

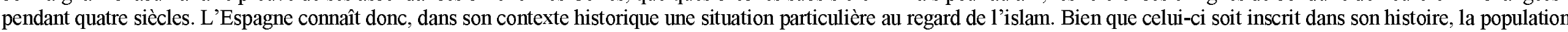
musulmane a longtemps été numériquement très faible sur son territoire, si l'on excepte les villes de Ceuta et Melilla, enclaves espagnoles sur le territoire marocain. 


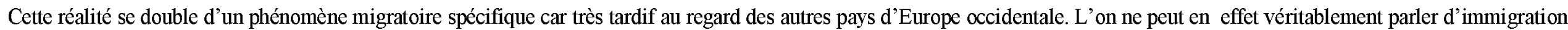

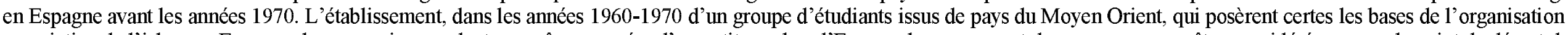

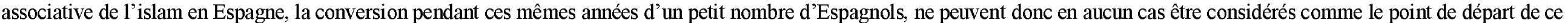

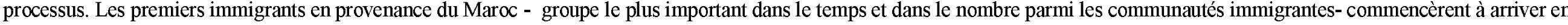

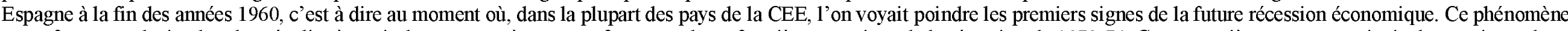

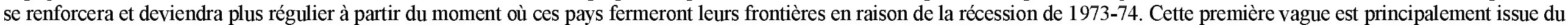
Maroc.

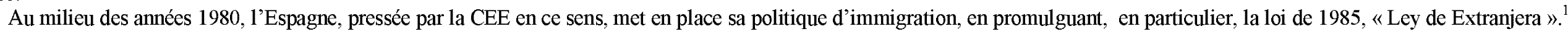

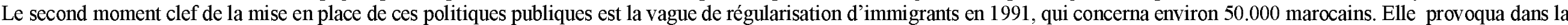
société espagnole un début de prise de conscience de l'importance que commençait à prendre les phénomènes migratoires en Espagne.

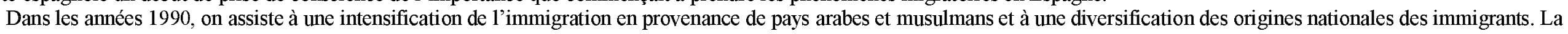
communauté marocaine demeure certes la plus importante numériquement, mais d'autres émergent, communauté algérienne, pakistanaise, sénégalaise ou nigeriane.

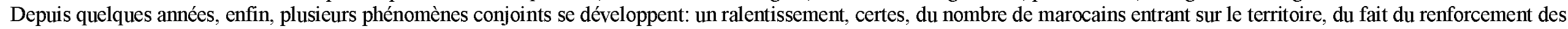

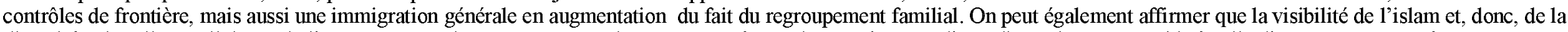
diversité culturelle et religieuse de l'Espagne va croisant. Pour autant, la communauté musulmane n'est pas d'une dimension comparable à celle d'autres pays européens.

\subsection{Composition nationale et données démographiques}

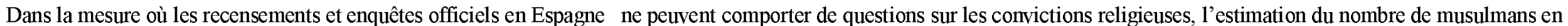

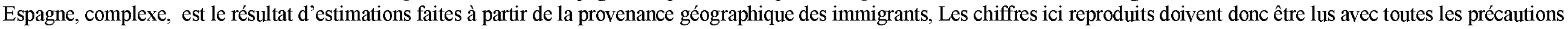
d'usage.

Estimation du nombre de musulmans étrangers en Espagne au $1^{\text {er }}$ janvier 2007

\begin{tabular}{lc}
\hline Marruecos & 582.923 \\
\hline Argelia & 45.813 \\
\hline Pakistán & 42.105 \\
\hline Senegal & 36.955 \\
\hline Nigeria & 32.119 \\
\hline Gambia & 17.393 \\
\hline Mali & 17.094 \\
\hline Mauritania & 9.271 \\
\hline Bangladesh & 6.480 \\
\hline
\end{tabular}

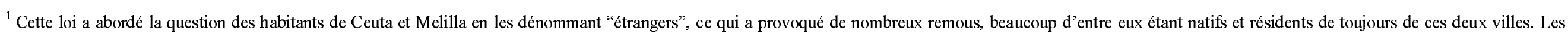
mobilisations qui en résultèrent contrainrent l'Etat à prendre les mesures nécesaires pour qu'ils puissent obtenir la nationalité espagnole. 


\begin{tabular}{lr} 
Egipto & 2.566 \\
\hline Irán & 2.334 \\
\hline Siria & 2.129 \\
\hline Turquía & 1.758 \\
\hline Túnez & 1.544 \\
\hline Líbano & 1.250 \\
\hline Jordania & 1.088 \\
\hline Sierra Leona & 989 \\
\hline Iraq & 880 \\
\hline \hline Total & $\mathbf{8 0 4 . 6 9 1}$ \\
\hline
\end{tabular}

Fuente: Instituto Nacional de Estadística (INE), 2007

On considère qu'à cette estimation du nombre de musulmans étrangers, il faut ajouter le montant des musulmans naturalisés, environ 100.000 personnes, et les Espagnols convertis à l'islam, qui représenteraient entre 1.000 et 5.000 personnes.

La Ucide, pour sa part, dans son Etude démographique de la population musulmane en Espagne, à partir des données du Recensement National 2007 et des données du Ministère de la Justice, établit les estimations suivantes ${ }^{2}$ :

${ }^{2}$ UCIDE, 2008, Estudio demográfico de la Población musulmana, Observatorio Andalusí. Le Recensement National est ici la référence fondamentale afin de cerner l'importance de la population musulamne de Ceuta et Melilla, et pour une connaissance détaillée de la population étrangère issue des 57 Etats membres de l'Organisation de la Conférence Islamique avec représentation permanente à l'ONU. Un calcul compensateur est appliqué afin de tenir compte des minorités immigrées en provenance de l'Europe et du continent américain, en particulier sud-américain. 


\begin{tabular}{|lr|}
\hline \multicolumn{1}{|c|}{ Ciudadanos españoles } & Muslimes \\
\hline Ceptíes (Habitants de Ceuta) & 28.070 \\
\hline Melillenses (Habitants de Melilla) & 30.036 \\
\hline Ceptíes y melillenses emigrados (Emigrés de ces deux villes) & 5.460 \\
\hline \hline Nacionalizados (naturalisés) & 80.235 \\
\hline Naturales ( originaires) & 16.020 \\
\hline Descendientes (descendants) & 168.861 \\
\hline Total de hispanomusulmanes & $\mathbf{3 2 8 . 6 8 2}$ \\
\hline
\end{tabular}


Zone d'origine des musulmans étrangers et nationalités

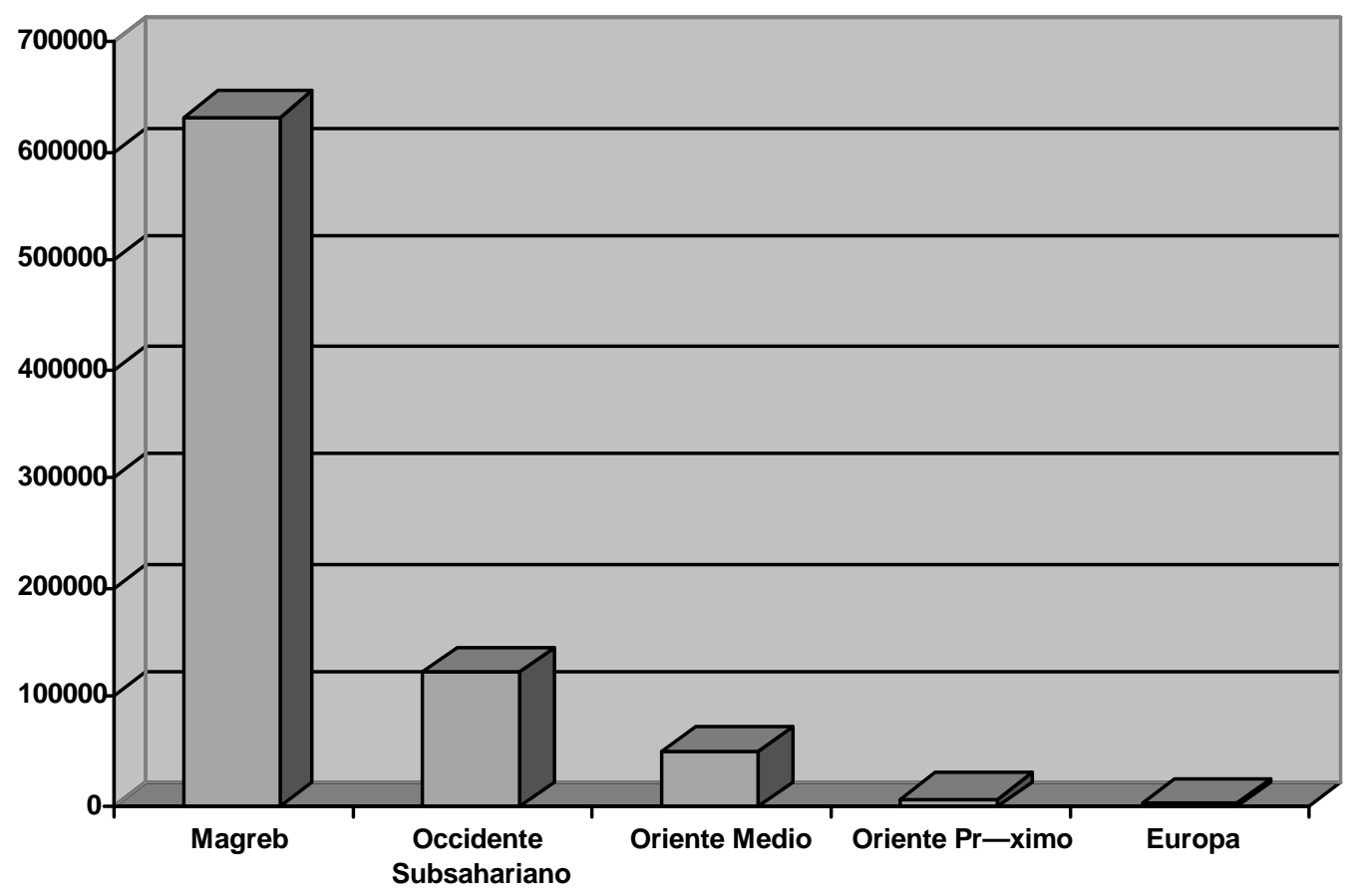




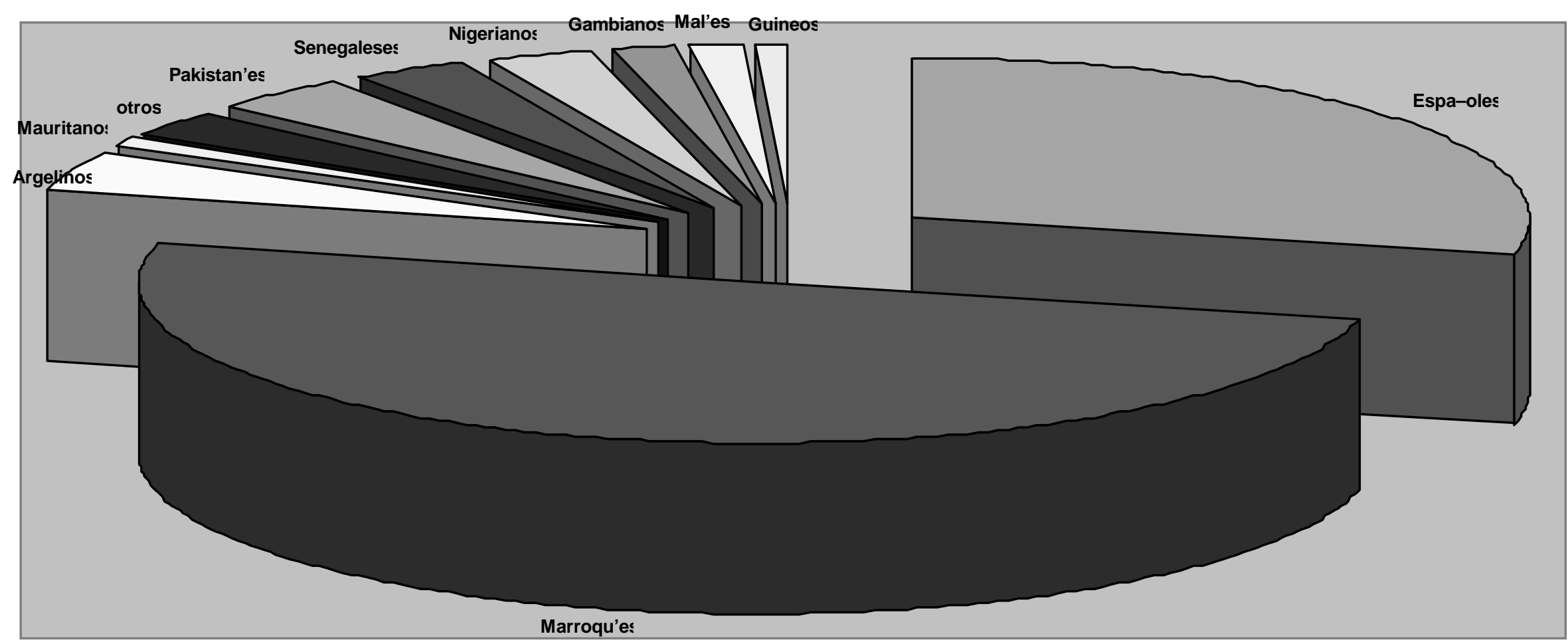




\section{Nationalité d'origine et Communauté Autonome de Résidence}

Ces statistiques ont été élaborées à partir des données du recensement national. Le nombre des étrangers munis d'un permis de travail et/ou de résidence, sont toujours sous-estimés, car l'on ne tient pas compte des mineurs ni des procédures en cours. Figurent également ici le nombre de communes par province qui permet d'apprécier la densité démographique de la population musulmane espagnole ou étrangère par Communauté. 


\begin{tabular}{|c|c|c|c|c|c|c|}
\hline Autonomías & Provincias & Municipios & Extr & jeros & Españoles & Totales \\
\hline \multirow{8}{*}{ Andalucía } & Almería & 102 & 42.032 & \multirow{8}{*}{109.073} & \multirow{8}{*}{75.357} & \multirow{8}{*}{184.430} \\
\hline & Cádiz & 44 & 6.728 & & & \\
\hline & Córdoba & 75 & 3.219 & & & \\
\hline & Granada & 168 & 9.660 & & & \\
\hline & Huelva & 79 & 7.162 & & & \\
\hline & Jaén & 97 & 5.912 & & & \\
\hline & Málaga & 100 & 26.091 & & & \\
\hline & Sevilla & 105 & 8.269 & & & \\
\hline \multirow{3}{*}{ Aragón } & Huesca & 202 & 5.444 & \multirow{3}{*}{25.189} & \multirow{3}{*}{5.793} & \multirow{3}{*}{30.982} \\
\hline & Teruel & 236 & 3.631 & & & \\
\hline & Zaragoza & 293 & 16.114 & & & \\
\hline Asturias & Asturias & 78 & 2.623 & 2.623 & 108 & 2.731 \\
\hline Baleares & Baleares & 67 & 25.178 & 25.178 & 681 & 25.859 \\
\hline \multirow{2}{*}{ Canarias } & Las Palmas & 34 & 17.472 & \multirow{2}{*}{23.802} & \multirow{2}{*}{30.834} & \multirow{2}{*}{54.636} \\
\hline & Santa Cruz & 53 & 6.330 & & & \\
\hline Cantabria & Cantabria & 102 & 2.098 & 2.098 & 81 & 2.179 \\
\hline \multirow{5}{*}{ Castilla La Mancha } & Albacete & 87 & 4.936 & \multirow{5}{*}{27.489} & \multirow{5}{*}{5.471} & \multirow{5}{*}{32.960} \\
\hline & Ciudad Real & 102 & 4.083 & & & \\
\hline & Cuenca & 238 & 2.527 & & & \\
\hline & Guadalajara & 288 & 4.145 & & & \\
\hline & Toledo & 204 & 11.798 & & & \\
\hline \multirow{9}{*}{ Castilla y León } & Ávila & 248 & 2.100 & \multirow{9}{*}{16.744} & \multirow{9}{*}{622} & \\
\hline & Burgos & 371 & 3.531 & & & \\
\hline & León & 211 & 2.948 & & & \\
\hline & Palencia & 191 & 656 & & & \\
\hline & Salamanca & 362 & 1.620 & & & 17.366 \\
\hline & Segovia & 209 & 2.134 & & & \\
\hline & Soria & 183 & 1.349 & & & \\
\hline & Valladolid & 225 & 2.002 & & & \\
\hline & Zamora & 248 & 404 & & & \\
\hline & Barcelona & 311 & 170.755 & & & \\
\hline Cataluña & Gerona & 221 & 45.570 & 274473 & 4564 & 279037 \\
\hline Galdunta & Lérida & 231 & 21.376 & 217.410 & 4.004 & 210.001 \\
\hline & Tarragona & 183 & 36.772 & & & \\
\hline Ceuta & Ceuta & 1 & 2.467 & 2.467 & 28.070 & 30.537 \\
\hline Eytromadura & Badajoz & 164 & 3.765 & 10028 & 5508 & 15536 \\
\hline Extremadura & Cáceres & 219 & 6.263 & $10.0<0$ & 5.500 & 15.000 \\
\hline & La Coruña & 94 & 2.195 & & & \\
\hline Galicia & Lugo & 67 & 867 & 6488 & २२1 & 6709 \\
\hline & Orense & 92 & 644 & & & \\
\hline & Pontevedra & 62 & 2.782 & & & \\
\hline La Rioja & La Rioja & 174 & 10.314 & 10.314 & 59 & 10.373 \\
\hline Madrid & Madrid & 179 & 95.582 & 95.582 & 101.107 & 196.689 \\
\hline Melilla & Melilla & 1 & 4.361 & 4.361 & 30.036 & 34.397 \\
\hline Murcia & Murcia & 45 & 62.314 & 62.314 & 726 & 63.040 \\
\hline Navarra & Navarra & 272 & 10.602 & 10.602 & 282 & 10.884 \\
\hline & Alicante & 141 & 37.716 & & & \\
\hline Valencia & Castellón & 135 & 17.308 & 91.800 & 38.671 & 130.471 \\
\hline & Valencia & 266 & 36.776 & & & \\
\hline & Álava & 51 & 5.442 & & & \\
\hline Vascongadas & Guipúzcoa & 88 & 4.163 & 16.117 & 491 & 16.608 \\
\hline & Vizcaya & 112 & 6.512 & & & \\
\hline 19 & 52 & 8.111 & 816 & & 328.682 & 1.145 .424 \\
\hline
\end{tabular}


Nous reprenonss ici les conclusions de l'enquête menée en septembre 2007 par l'Institut Metroscopia pour le compte des Ministères de l'Intérieur, de la Justice du Travail et des Affaires Sociales ${ }^{3}$.

Cette étude établit tout d'abord que la quasi-totalité des membres de la communauté eserce une activité économique, ce qui la différencie à la fois de l'ensemble de la société et de l'ensemble des étrangers en Espagne. Le taux d'activitét ${ }^{4}$ des immigrants musulmans de plus de 16 ans s'élèverait en effet à $86 \%$, soit $29 \%$ de plus que la communauté nationale (57\%) et 10 de plus que l'ensemble des étrangers (76\%). Ce constat trouve sa principale explication dans la faible présence dans cette communauté d'étudiants et de femmes (chez ces dernières, le taux d'activité est plus faible). Trois immigrants sur 4 sont en effet des hommes et $90 \%$ d'entre eux sont âgés de moins de 45 ans.

Dans le même temps, le chomage declare est plus important que dans l'ensemble de la population espagnole : le taux de chômage au sein de la communauté musulmane est en effet de $22 \%$, contre $7 \%$ pour la population espagnole et $12 \%$ pour l'ensemble des étrangers.

Les données obtenues permettent également de faire ressortir un fort indice de précarité, un immigrant musulman sur 4 seulement affirmant travaillerer avec un contrat à durée indéterminée, deux tiers se voyant contraints de travailler avec des contrats à durée déterminée, et $18 \%$ de manière illégale.

En matière d'activité professionnelle, les immigrants musulmans occupent, par rapport aux travailleurs espagnols, des postes à moindre qualification et aux niveaux les plus bas du marché du travail. $26 \%$ travaillent en tant qu'ouvrier spécialisé, $20 \%$ comme ouvrier non spécialisé, $14 \%$ comme personnel non qualifié, $13 \%$ comme vendeur ou employé. $10 \%$ se déclarent commerçant avec des employés. Il faut néanmoins signaler qu'il existe un nombre non négligeable d'exceptions à ces tendances. Dans le domaine agricole, en revanche, la tendace générale est confirmée, les immigrants musulmans étant avant tout des tavailleurs agricoles au service de propriétaires nationaux. Quant aux commerçants, il apparait que dans la majorité des cas, ils tienent un type de commerce (bars, alimentation, cartes et appels téléphoniques) fournissant quasi exclusivement des biens et services aux members de leur propre communauté, biens et services non pris en charge par le commerce national traditionnel.

En matière éducative, enfin, le neveau de formation est faible, l'absence d'études et l'illettrisme étant trois fois plus élevés que dans la société dans son ensemble. Plus d'un tiers des immigrants musulmans a quitté le système scolaire avant d'y avoir accompi les dix ans habituels, $9 \%$ seulement bénéficient d'une formation universitaire, et $4 \%$ ont poursuivi leur parcours dans le système universiatire au delà des premiers degrés.

\subsection{Cadre légal : la reconnaissance institutionnelle de l’islam}

L'Espagne, depuis la mise en place de la Constitution de 1978, est marquée par l'autonomisation de l'Etat des Églises, particulièrement de l'Eglise catholique, et de la religion, rompant ainsi avec la presque totalité de son histoire. Ce phénomène se traduit par l'attribution à tous les Espagnols de l'ensemble des droits civils et politiques, sans discrimination aucune, et singulièrement sans discrimination religieuse. La démocratisation voit donc dans ce pays la naissance d'une véritable citoyenneté démocratique.

La religion musulmane bénéficie, au sein de ce cadre qui n'a pas souhaité traduire les principes de liberté et de neutralité par une forme séparative, d'une reconnaissance institutionnelle. Ce processus d'intégration juridique objective a connu plusieurs étapes :

-d'une part, le vote de la Constitution de 1978, avec 1' article 16 alinéa 1, assurant les libertés idéologique, religieuse et de culte et l'article 16 alinéa 3 prévoyant la signature d'accords de coopération avec les grandes religion minoritaires du pays, celles qui, par leur cadre et nombre de croyants, peuvent se prévaloir d'un enracinement notoire, notorio arraigo, en Espagne ; - le vote de la Loi sur la Liberté Religieuse du 5 juillet 1980 (LOLR), qui constitue le développement normatif de l'article 16 de la Constitution, et précise les modalités de ces accords ; elle déclare également dans son article 1 alinéa 1 que l'Etat garantit le droit fondamental à la liberté religieuse, et détaille, dans son article 2-1, le contenu de celle-ci concernant le domaine des croyances : La LOLR prévoit enfin dans ses article 5 et 6 que les Eglises, confessions et communautés religieuses auront la personnalité juridique une fois inscrites sur un registre spécifique au Ministère de la Justice. L'inscription implique pour les bénéficiaires une pleine autonomie et la possibilité d'établir leurs propres normes d'organisation, régime interne et régime du personnel ;

-1'accès, conformément à ce texte à la reconnaissance légale. Le 21 février 1990 voit la signature d'accords entre l'Etat et les représentants de l'islam, mais aussi du judaïsme et du protestantisme, pour définir les conditions d'exercice de la liberté religieuse et préciser le statut des lieux et ministres du culte, garantissant toute liberté en ce domaine. Ils ouvrent également la possibilité d'aumôneries et de la mise en place d'un enseignement confessionnel.

\footnotetext{
${ }^{3}$ Metroscopia: "Musulmanes de España. Segunda oleada del Estudio de Opinión a población musulmana de origen inmigrante", en http://www.mir.es/EDSE/informe_musulmanes.pdf

${ }^{4}$ Ceci signifie qu'ils travaillent ou sont en recherche d'un emploi.
} 
-la signature d'un accord de coopération tel que prévu par l'article 7 de la LOLR en 1992, qui a impliqué deux éléments. Le premier a été la nécessité d'une réorganisation interne de l'islam espagnol puisque la loi imposait un interlocuteur unique. Furent créées en 1989 la Fédération des Entités religieuses islamiques, FEERI, Federación Española de Entidades Religiosas Islámicas et, en 1991, l'Union des Communautés Islamiques, UCIE, Unión de la Comunidades Islámicas de España. La plus grande partie des communautés et associations islamiques espagnoles se sont structurées autour de ces deux fédérations. Celles-ci se sont regroupées en 1992 pour constituer la Commission Islamique d'Espagne, CIE, Comisión Islámica de España, interlocuteur de l'Etat ${ }^{5}$.

La signature de l'accord de coopération a également impliqué reconnaissance à l'islam du caractère d'enracinement notoire en Espagne, qui a eu lieu en 1989. On y incluait divers facteurs renforçant l'idée de l'importance de l'islam en Espagne : le nombre de fidèles et l'ancienneté de la confession, la proximité géographique avec le monde musulman qui favorise l'accroissement d'une confession déjà enracinée dans le pays, et l'existence d'une immigration en provenance de pays musulmans. L'accord renforce la liberté effective du culte en légalisant le respect du jour de repos religieux, ouvrant la possibilité de dates alternatives dans les centres d'enseignement public et privés et pour les concours d'entrée dans les administrations publiques. Il accorde également une liberté d'exercice religieux dans l'armée et permettent la création d'aumôneries dans les hôpitaux et prisons.

Il établit dans ses diverses dimensions, pour la première fois depuis cinq siècles, la présence de l'islam dans la vie publique espagnole au même niveau que les autres confessions religieuses .

\subsection{Reconnaissance et intégration}

Ainsi, pour la minorité musulmane, comme pour l'ensemble de la population, l'intégration légale est acquise. De plus, un travail conscient de réintégration dans la communauté nationale, de la part des acteurs institutionnels, se déploie depuis 1978.

Comme nous venons de le voir, l'intégration légale comprend déjà une dimension symbolique, de représentation. Celleci, et les actions déployées en ce sens depuis la démocratisation ont fait l'objet de nombreuses études ${ }^{7}$. Nous souhaitons ici, à titre d'illustration, évoquer un moment représentatif, l'année 1992. Cette année, 500aire d'une date capitale pour l'Espagne, a débuté avec la première visite en Espagne d'un président d'Israël. Elle a vu la commémoration de l'édit d'expulsion des juifs de 1492, avec les cérémonies Sefarad 92, et les cérémonies Al Andalus 92, axées sur la célébration de la richesse de la civilisation arabo-musulmane. Le 4 novembre, dans un discours prononcé à Cordoue, le roi a également insisté sur la coopération entre l'Espagne et les pays du monde arabe, dans un esprit de recherche de consensus et dans une présentation de la nouvelle Espagne moderne et démocratique, soulignant ainsi que les affrontements entre Islam et chrétienté appartenaient à un passé révolu. Plus généralement, tous les discours tenus par l'Etat et ses représentants ont cherché à créer un consensus autour des idées phares de démocratie et de modernité.

Plus récemment, dans le but de renforcer l'intégration légale, symbolique, mais aussi réelle des différentes minorités religieuses en Espagne, en rendant plus effectives les conditions de l'égalité, le gouvernement de José Luis Rodriguez Zapatero a souhaité mettre en place une fondation d'Etat reliée au Ministère de la Justice, qui a vu le jour le 25 janvier 2005, la fondation Pluralismo y Convivencia, Pluralisme et Vivre Ensemble. Le but de cette fondation est que « les religions minoritaires acquièrent plus de visibilité pour la société espagnole et que les centres religieux soient des lieux d'intégration, afin de prouver et rendre effective la compatibilité de la liberté de croyances avec les valeurs constitutionnelles qui sous-

\footnotetext{
${ }^{5}$ Cette construction d'un interlocuteur unique ne fut certes pas chose aisée. Elle a nécessité la réorganisation d'un mouvement associatif très dispersé, reflet de la composition hétérogène de la communauté. Historiquement, la première assocation musulmane nationale, association culturelle, date de 1971. Elle avait pu voir le jour à la faveur d'une loi du régime franquiste finissant autorisant le développement d'autres croyances sous réserve qu'elles respectent le catholicisme et l'Eglise catholique.

Elle avait été précédée, compte tenu de la situation particulière de Melilla, de l'apparition d'une première association sur le territoire espagnol dans cette ville

Dans la Péninsule, la première association inscrite au registre des Entités Religieuses du Ministère de la Justice fut l'Association Musulmane d'Espagne, Asociación Musulmana en España (23 avril 1971), présidée par Riay Tatary Bakry, actuel président de la CIE. Cette association obtint de construire la première mosquée de la capitale. C'est à la faveur de la Transition, enfin, que l'on assista au développement de groupes de convertis espagnols, tout d'abord, puis de groupes liés aux phénomènes migratoires, encore balbutiants. A partir du milieu des années 1980, enfin, apparaissent des associations cherchant à encadrer les nouvelles populations immigrées qui s'installent en Espagne, dans lesquelles, souvent, s'expriment les intérêts de certaines ambassades de pays arabes et musulmans.

Les sensibilités différentes qui persistent dans les deux fédérations constituant la CIE générent parfois d'importantes discussions sur les modalités de développement de certains aspects de l'accord de coopération de 1992. Il en a été ainsi, nous y reviendrons, pour la question du contenu de l'enseigement de la religion musulmane à l'école publique.

${ }^{6}$ Le Real Decreto 710/2006 de 9 de junio, décret du 9 juin 2006, constitue le développement des Accords de coopération dans ce domaine. Il est consacré à l'assistance religieuse et au respect de la liberté de culte en milieu carcéral. Il prévoit entre autres que l'assistance sera dispensée par des ministres du culte, rémunérés pour ce faire, désignés par les communautés et autorisés par l'administration pénitentiaire compétente, l'autorisation ayant une validité d'un an, renouvelable. Des bénévoles pourront également remplir ces missions, sous réserve du respect des conditions énoncées dans le décret.

${ }^{7}$ On se reportera par exemple à Vincent, Bernard 1995. 1492, L'année admirable. Flammarion, Paris
} 
tendent la Démocratie. ${ }^{8}$ Le gouvernement a souhaité, pour ce faire, « contribuer à l'exécution de programmes et projets de caractère culturel, éducatif et d'intégration sociale des confessions non catholiques ayant passé un Accord de coopération avec l'Etat espagnol ou d'enracinement notoire en Espagne. ${ }^{9}$ " Trois millions d'euros ont été, dans ce but, affectés à cette fondation par 1'Etat. Si les accords de 1992 passés avec l'Etat avaient ouvert la possibilité de financement des minorités concernées, aucune action en ce sens n'avait vu le jour avant la création de cette Fondation. Or, la différence de situation d'avec 1'Eglise catholique, dont le financement ressort pour une part non négligeable du budget de l'Etat, et qui se voit octroyer la part de l'impôt sur le revenu des personnes s'étant déclarées en ce sens, ne pouvait que renforcer le sentiment d'inégalité et de marginalisation des autres religions, dont l'islam, financées uniquement par les dons des fidèles.

Les activités ici concernées sont, en particulier, des projets visant à l'installation de centres culturels, à la formation du personnel religieux, à l'établissement de programmes pour l'enseignement, mais aussi des activités d'entraide sociale. Concernant la formation de personnels religieux, la question, longtemps laissée de côté, a été soulevée de manière plus aiguë après les attentats de mars 2004 à Madrid, puisque les enquêtes ont établi l'existence de liens entre certains terroristes et des imams de mosquées clandestines en Espagne. C'est pourquoi le gouvernement a réalisé et affirmé la nécessité d'une mise en place d'une régulation du culte, et du développement de moyens nécessaires en ce sens, en particulier en matière de formation institutionnelle des imams. Il faut enfin noter que tout financement du culte est exclu, et que l'action menée ici par l'Etat a pour objectif, à moyen terme, de parvenir à un autofinancement total de toutes les confessions religieuses.

L'on peut donc considérer que l'Espagne, vis-à-vis de l'islam, occupe une position spécifique, et ce pour deux raisons : -la singularité de la présence musulmane, tout d'abord, issue de plusieurs sources, trajectoire d'immigration depuis les années 1970 surtout, mais aussi présence particulière d'une communauté interne, particulièrement au travers des deux villes de Ceuta et Melilla, sans oublier la trace de la place de l'islam dans l'histoire et la culture de ce pays ;

-la mise en place d'un cadre de reconnaissance légale et institutionnelle très structuré et très étendu.

Or, en dépit de ces éléments objectifs, et en dépit des actions légales et symboliques entreprises par l'Etat, il apparaît relativement évident que la question de l'islam en Espagne est une question qui continue à être posée sur un mode problématique $^{10}$. Ceci est la première constatation qui peut nous guider pour esquisser un jugement sur la manière dont sont considérés les formes et degrés d'intégration des musulmans en Espagne.

Le caractère problématique évoqué ci-dessus est à imputer à diverses raisons, dont la principale tient à une vision de l'islam comme facteur externe à la société espagnole. Ce point de vue est aussi présent concernant les musulmans de nationalité espagnole, qui dénoncent, dans leurs discours ${ }^{11}$, la thématique du maure étranger, foráneo, un veneno educado , un venin transmis par l'éducation. Il s'agit du reflet d'une préoccupation très importante, celle de la conscience que l'islam et les musulmans sont vécus par une proportion non négligeable d'Espagnols comme étrangers, entes extráneos, extérieurs à l'hispanité et à l'Espagne, qu'il y a là incompatibilité, voire même danger pour le modèle social dominant et ceci malgré la présence de tous les témoignages de la présence historique de l'islam en Espagne. En témoignent les propos, jamais réfutés officiellement, du général Manglano, Chef du Service d'Information de la Défense, et donc haute autorité militaire, à un journaliste de la revue Panorama en 1992, qui déclarait que les musulmans d'Espagne sont la cinquième colonne du monde arabe et islamique en Espagne, et ceci, quelques jours avant la signature de l'Accord de Coopération CIE-Etat espagnol.

Quelles sont les causes fondamentales de ce phénomène ? Elles seraient à rechercher, selon les musulmans espagnols, dans l'histoire. L'Espagne s'est faite contre l'islam (comme contre le protestantisme ou le judaïsme). Cette lutte a entraîné préjugés et ignorance, et ancré l'idée du moro traidor, du maure traître, même si le maure étranger fut aussi le maure indispensable, imprescindible, à la réussite de la fusion des peuples espagnols.

Selon les musulmans espagnols, pour beaucoup d'Espagnols, être musulman signifie être arabe, ce qui implique une incompatibilité totale entre islam et hispanité, opinion basée sur la confusion de critères religieux et ethniques. Ceci est pour eux lié à une ignorance fondamentale de l'histoire et des caractéristiques de l'islam en général et de l'islam espagnol en particulier, phénomène résultant également d'une longue et nécessaire clandestinité ayant entraîné une sorte de culture de la survie par le secret. Il faut noter que, face à ce contexte, les musulmans espagnols, s'ils définissent leur hispanité de diverses

\footnotetext{
${ }^{8}$ Communiqué du Ministère de la Justice, 5 octobre 2004. Voir "El Gobierno exige la autofinanciación de todas las confesiones religiosas", $A B C, 6$ de octubre de 2004 ; "El Gobierno no financiará la práctica del culto musulman", El País, 6 de octubre de 2004 ; "Religiones minoritarias recibirán mas ayuda", El Periódico, 6 de octubre de 2004.

${ }^{9}$ Texte de l'arrêté de création du 8 mars 2005.

${ }^{10}$ Nous nous appuyons ici, entre autres, sur les analyses de Jordi Moreras, par exemple dans "Lógicas divergentes. Configuración comunitaria e integración social de los colectivos musulmanes en Cataluña", www.equintanilla.com/Dossier/Logicas\%20divergentes\%20Moreras.pdf

${ }^{11}$ Nous reprenons ici les conclusions de travaux personnels, ayant donné lieu à une communication au Congrès de la Société Internationale de Sociologie des Religions en juillet 2007 à Leipzig, Modernité, identité nationale et religion: la reconstruction de la mémoire et la minorité musulmane dans l'Espagne démocratique.

Ils sont basés sur le dépouillement systématique depuis sa création, fin 1994, jusqu'en 2004 d'un des rares journaux confessionnels musulmans espagnols, Verde Islam, journal en ligne de la FEERI, une des deux composantes de la CIE. D'accès direct, il relève plus généralement du site webislam, un des seuls sites en langue espagnole sur l'islam et les musulmans espagnols, fait par ceux-ci.
} 
manières, selon divers critères, plus culturels ou plus civiques selon les cas, ne la revendiquent pas, car, précision que l'on rencontre souvent dans leur discours, elle est : « Nous, citoyens espagnols de religion musulmane, (...), nous ne revendiquons pas l'hispanité (de notre projet) ; nous sommes espagnols. $»^{12}$

Le caractère de "facteur extérieur », présent dans certains discours, paraît bien évidemment fort éloigné des mots reconnaissant la condition d'enracinement notoire à l'islam. Cette contradiction se retrouve jusque dans les propos officiels du Ministère de la Justice, qui dans le Guide des Entités Religieuses (Guia de Entitades Religiosas de España), édité par la Direction Générale des Affaires Religieuses (Dirección General de Asuntos Religiosos) en 1998, affirmait : "L'islam est en passe de devenir la confession minoritaire la plus importante numériquement en Espagne, même si elle demeure une confession de 'service' (de servicio) à une population immigrée. »

Le « facteur extérieur », pour reprendre l'expression de Jordi Moreras ${ }^{13}$, procède en outre, en ce qui concerne cette fois la présence musulmane issue de l'immigration ${ }^{14}$ du fait que cette dernière est considérée avant tout par les medias, l'opinion publique, voire les acteurs publics ou parfois la communauté scientifique, à travers une seule clef de lecture, id est l'immigration. Par conséquent, les situations liées à la présence de l'islam en Espagne sont exclusivement traduites en termes de problèmes et difficultés liées à l'immigration.

Il nous faut également mentionner le fait que les attentats du 11 septembre, et la stigmatisation de l'islam et des musulmans qui en a résulté, ont encore renforcé le caractère problématique dans cette perception, et ceci pour trois séries de raisons. Tout d'abord, l'islam a été considéré comme un facteur de conflictualité sociale, affirmation à l'appui de laquelle on cite l'hostilité enregistrée chez les habitants de différentes villes à l'apparition ou à la construction de lieux de culte. Puis, l'islam a été vu comme un frein au processus d'intégration sociale initiés par certaines communautés immigrées, argument souvent basé sur l'idée sous-jacente que cette intégration ne pouvait se réaliser que par une assimilation. La question qui, en réalité, résume l'état du débat, et qui peut ête considérée comme une des questions les plus prégnantes aujourd'hui est alors : les expressions d'identité ou de confession musulmane sont elles un obstacle rédhibitoire à l'intégration de ces communautés ? ${ }^{15}$ Selon les résultats des enquêtes européennes menées par les organismes officiels de 1'Union de surveillance de la discrimination et de la xénophobie, 65 pour cent des Espagnols considèrent que les musulmans souhaitent rester distincts de 1 'ensemble de la population ${ }^{16}$, et $58 \%$ qu'il existe un conflit entre être un musulman dévot et vivre dans une société moderne $^{17}$. Ces mêmes enquêtes montrent, à l'inverse, le succès des syndicats dans leur volonté de rendre les entreprises plus flexibles au regard des exigences découlant des différences culturelles et religieuses de leurs employés ${ }^{18}$.

Enfin, quelques conflits ont fait resurgir à la rentrée 2007 la question du port du voile en milieu scolaire. Dans ces différentes situations, l'interdiction figurant dans le règlement intérieur des établissements incriminés et qui a conduit au renvoi des jeunes filles ou fillettes a été dénoncée par les autorités scolaires locales, avec obligation de réintégration, au nom du droit à l'éducation et de la priorité de la scolarisation. La question qui demeure en débat dans la société, tant au niveau des responsables d'établissement, des parents d'élèves que des politiques, est celle de la nécessité ou de l'inutilité d'une réglementation générale Celle-ci est évidemment reliée au délicat problème de la signification de l'aconfessionnalité de l'Etat espagnol, qui garantit à chacun le libre choix de ses convictions et, plus avant, des limites de la liberté religieuse Le premier gouvernement Zapatero, qui a pris fin en mars 2007, avait indiqué, et ceci semble être l'opinion d'une majorité, qu'il ne souhaitait pas mettre en œuvre une législation générale, accordant sa préférence au règlement au cas par cas ${ }^{19}$.

\footnotetext{
${ }^{12}$ Verde Islam, 1997.

${ }_{14}^{13}$ Moreras, J, op cit.

${ }^{14}$ Les deux populations peuvent, bien entendu, se recouper, la distinction que nous avons établie est de caractère analytique. Une partie des musulmans de nationalité espagnole sont des personnes d'origine étrangèrs ayant obtenu la nationalité espagnole. (On se référera aux estimations cidessus). Notons qu'un immigré de la première génération sur dix avait obtenu, en octobtre 2007, la nationalité espagnole.

${ }^{15}$ Cette question ne relevant pas directement du thème de ce passage, l'on se reportera à Moreras, J., op cit et Proeschel, C., op cit, qui montrent le premier pour des communautés d'immigrés, la seconde pour les musulmans de nationalité espagnole, comment ces deux aspects d'islamité et

d'intégration sont complémentaires et non antagonistes, et comment l'expression d'une identité musulmane peut constituer un facteur nécessaire et facilitant de cette intégration.

${ }^{16}$ Pew Global Attitudes Project, Public Opinion Survey, May 2005 Report.

${ }^{17}$ Pew Global Attitudes Project, Public Opinion Survey, June 2006 Report.

${ }^{18}$ On consultera ici Pin, J.R. (eds). 2004. Libro Blanco sobre las mejores prácticas para la integración del trabajador inmigrante en las empresas españolas, IESE, Creade, Sagardoy Abogados, Pamplona.

${ }^{19}$ Pour une vue plus précise des différents courants d'opinion s'étant exprimés :

http://medios.mugak.eu/noticias/noticia/115178, http://www.equintanilla.com/Dossier/velo\%20efecto\%201lamada.pdf)
} 


\section{Enseignement et religion}

\subsection{L'enseignement de la religion à l'école publique : situation générale}

Il apparaît nécessaire ici d'exposer les principales lignes de l'article 27 de la Constitution de 1978, consacré à l'éducation, afin de développer ses conséquences en matière d'enseignement de la religion à l'école publique et la manière dont elles sont traduites à la fois dans les accords avec les confessions, ici la confession musulmane et dans les lois scolaires.

L'article 27 de la Constitution énonce :

1 Tous ont droit à l'éducation. La liberté d'enseignement est reconnue.

2-L'éducation aura pour objet le plein épanouissement de la personnalité humaine, dans le respect des principes démocratiques de vie en commun et des droits et libertés fondamentales.

3-les pouvoirs publics garantissent le droit des parents à ce que leurs enfants reçoivent la formation religieuse et morale en accord avec leurs propres convictions.

$[\ldots]$

5-les pouvoirs publics garantissent le droit de tous à l'éducation(...)

$[\ldots]$

La philosophie générale de ce texte est claire : l'éducation, base de l'épanouissement de la personne, est un droit au même titre que les autres droits fondamentaux édictés dans la Constitution, et doit être développée dans le respect de ceux ci, en particulier les droits en matière de liberté idéologique et de croyance. Tous les citoyens ayant droit à une éducation en ces termes, il appartient à l'Etat d'en assurer la possibilité. Or, dans la philosophie du nouvel Etat, pluraliste et démocratique, de cette obligation de l'Etat doivent découler des droits : celui de chacun de choisir le type d'éducation qu'il souhaite et de le mettre en conformité avec ses croyances, ainsi que le droit de chaque élève de recevoir la formation spirituelle en accord avec ses propres convictions. Et, en régime de coopération, il appartient à l'Etat d'assurer cette possibilité d'exercer sa liberté de conscience à l'intérieur de l'école publique.

Le droit et la possibilité pour les élèves de recevoir dans les établissements publics (et privés) un enseignement religieux en accord avec leurs convictions est garanti tant par l'accord avec le Saint-Siège sur l'Enseignement et les Affaires Culturelles que par les trois accords de coopération. Il l'est aussi par les différentes lois scolaires. Les religions pouvant être enseignées, en théorie, à l'école publique sont donc la religion catholique et les trois grandes religions minoritaires. Cependant, dans ce système de " cultes reconnus », cette mesure bénéficie surtout à l'Eglise catholique, du fait de son implantation officielle plus ancienne, du caractère récent des convenios et de leurs difficultés d'application. Nous y reviendrons à propos de l'islam.

Enfin, les professeurs et programmes sont proposé et élaborés par les instances religieuses, et désignés par les autorités académiques.

Les débats, qui se sont amplifiés depuis quelques années, portent à la fois sur le caractère obligatoire de la matière religion, sur sa prise en compte dans l'évaluation des élèves, mais aussi sur la nature d'une matière alternative.

Pendant toute la période de gouvernement socialiste (1982-1996), si l'enseignement de la religion catholique se devait d'être offert par les écoles publiques, cette matière était une option pour les élèves, et n'entrait donc pas en compte dans leur évaluation.

La Ley sobre la Calidad de la Enseñanza du 23 décembre 2003, texte très controversé, voté sous le gouvernement de droite du Partido Popular, au pouvoir entre 1996 et 2004, a voulu, au contraire, conférer valeur académique et obligatoire à cette matière, en réponse aux préoccupations exprimées par les évêques sur la déchristianisation de la société espagnole. Cette loi a mis en place une nouvelle matière, intitulée Société, Culture et Religion, comprenant deux versions, une de caractère confessionnel (catholique), et une de caractère non confessionnel, mais considérée par la majorité des partis progressistes comme oeuvre déguisée de l'épiscopat. Ces deux matières, obligatoires, faisaient donc l'objet d'une notation intervenant dans l'évaluation scolaire des élèves.

Cependant, après la défaite du Partido Popular en mars 2004, et l'arrivée au pouvoir du gouvernement socialiste dirigé par José Luis Zapatero, l'application de cette réforme a été gelée. Le nouveau gouvernement souhaitait le retour à l'ancien statut, optionnel, et sans statut académique, ainsi que la création d'une nouvelle matière alternative, relative aux valeurs civiques.

Ces différents souhaits ont été traduits dans une nouvelle loi scolaire, la dernière en date, la Loi Organique sur l'Education, Ley Orgánica de Educación (Ley Orgánica 2/2006) entrée en vigueur le 3 mai 2006. Elle est développée par le Décret Royal (Real Decreto) 1513/2006 du 7 décembre 2006 « par lequel sont établis les enseignements minimaux de l'éducation primaire» et par le décret Royal 1631/2006 du 26 décembre 2006 " par lequel sont établis les enseignements minimaux de l'éducation secondaire obligatoire ». Des principes importants sont ici énoncés :

-La religion est une matière optionnelle. Les établissements d'enseignement sont tenus d'offrir cette option ${ }^{20}$. Ils doivent également proposer une matière alternative pour les élèves ne désirant pas suivre cette option.

-Cette option pourra concerner la religion catholique ou toute autre religion ayant signé un accord de coopération avec l'Etat.

\footnotetext{
${ }^{20}$ Cet enseigenment est déclaré par la loi "de oferta obligatoria para los centros y de carácter voluntario para los alumnos".
} 
-Son évaluation sera identique à celle des autres matières. En revanche, les notes obtenues ne seront pas prises en compte dans l'élaboration des dossiers académiques ou des moyennes intervenant dans des processus d'admission où une sélection entre les élèves intervient.

-L'élaboration des programmes et cursus sera du ressort des autorités religieuses de chaque confession concernée.

\subsection{L'enseignement de l'islam à l'école publique}

Le droit des élèves à recevoir une éducation musulmane à l'école est détaillé dans l'article 10 de l'accord de coopération de 1'Etat avec la Comisión Islámica de España d'avril 1992. A cette date, deux domaines essentiels sont demeurés en suspens : la détermination du contenu de la matière, ainsi que le régime des professeurs chargés de l'enseigner. Les deux questions furent résolues quatre ans plus tard.

En janvier 1996, fut publié le « cadre des matières de l'enseignement islamique dans les établissements publics et sous contrat ». La discussion sur le contenu de cette matière avait en particulier trouvé son origine dans les différences de conceptions s'exprimant entre les deux entités constituant la CIE. La FEERI poursuivait deux objectifs explicites : établir d'une part un programme ouvert, accessible et attractif pour les élèves non musulmans et, d'autre part, considérer l'enseignement de l'islam non pas seulement comme l'enseignement des rites et principes de cette religion, mais aussi comme un enseignement fondé sur la mise en perspective d'aspects philosophiques et historiques. La UCIDE, pour sa part, souhaitait un enseignement recentré sur la confession religieuse. Il en résulta un cadre très, voire trop ample, adaptable certes aux besoins de chaque école, mais qui pâtit certainement de l'absence de matériaux spécifiques. Une des obligations des enseignants est dans ce texte l'amélioration des compétences des élèves en langue arabe, pour faciliter leur accès aux sources de leur religion et pour faciliter l'apprentissage de cette langue à l'extérieur de l'école.

A partir de janvier 2005, la CIE entama une refonte du programme d'enseignement, qui aboutit en 2006 à la publication du manuel de textes destiné à l'enseignement primaire Descubrir el islam, issu de la collaboration entre la UCIDE et la Fondation Pluralismo y Convivencia. Il constitue le premier ouvrage d'enseignement de la religion musulmane publié en Europe. Seul le recueil destiné à l'enseignement primaire est actuellement publié, les volumes suivants concerneront les autres niveaux du premier cycle. ${ }^{21}$ Cette publication participe certainement au développement des dispositions éducatives prévues dans l'Accord de 1992. Son objectif est également de favoriser les rencontres interculturellles et le dialogue religieux, en replaçant, dans la présentation, les valeurs de l'islam dans le contexte socioculturel espagnol. En 2007 ce matériel éducatif sera utilisé par les élèves de Ceuta, Melilla, l'Aragon et l'Andalousie, puis, dans les prochaines années, étendues à d'autres Communautés Autonomes.

En mars 1996, fut également signé l'accord sur la désignation et le régime économique des personnes chargées de l'enseignement de la religion musulmane dans les centres d'éducation primaire et secondaire ; la désignation des professeurs reste entre les mains de la Comisión et leur rémunération est assurée par l'Etat s'il y a au moins 10 élèves suivant cet enseignement dans l'école concernée. Pour ce qui concerne le mode de sélection des enseignants, les deux composantes de la CIE sont en accord sur les critères exigés : il doit s'agir de personnes musulmanes avec un bon niveau linguistique tant en espagnol qu'en arabe, niveau plus élevé pour les enseignants du niveau secondaire.

Pour autant, la question n'a toujours pas été résolue de manière satisfaisante, en particulier à Ceuta et Melilla ${ }^{22}$.On évalue, pour l'ensemble du territoire à $90 \%$ le pourcentage d'élèves de confession musulmane ne disposant pas de classe de religion $^{23}$. L'enseignement $n^{6}$ 'est pas toujours, comme pour la religion catholique, « mis à disposition », mais doit faire l'objet d'une demande de mise en place par les parents. On recense pour la dernière année scolaire 41 professeurs de religion musulmane à l'école publique alors que 314 étaient prévus pour véritablement mettre en œuvre cette enseignement et répondre aux besoins exprimés ${ }^{24}$.

\section{Evolution du nombre d'enseignants}

\footnotetext{
${ }^{21} \mathrm{http} / /$ prensa.grupo-sm.com/2006/10/descubrir_el_is.html

http://prensa.grupo-sm.com/files/DossierDescubrirelIslam.pdf

${ }^{22}$ Le fort taux de population musulmane à Ceuta et Melilla entraîne une demande bien plus importante en matière d'enseignement de l'islam à l'école publique. Ainsi, environ $60 \%$ des élèves des écoles primaires seraient de confession musulmane.

${ }^{23}$ Ce chiffre est issu de UCIDE, 2008. Estudio demográfico de la Población musulmana, Observatorio Andalusí,

${ }^{24}$ Chiffres officiels de la CIE.
} 


\section{Contratación}

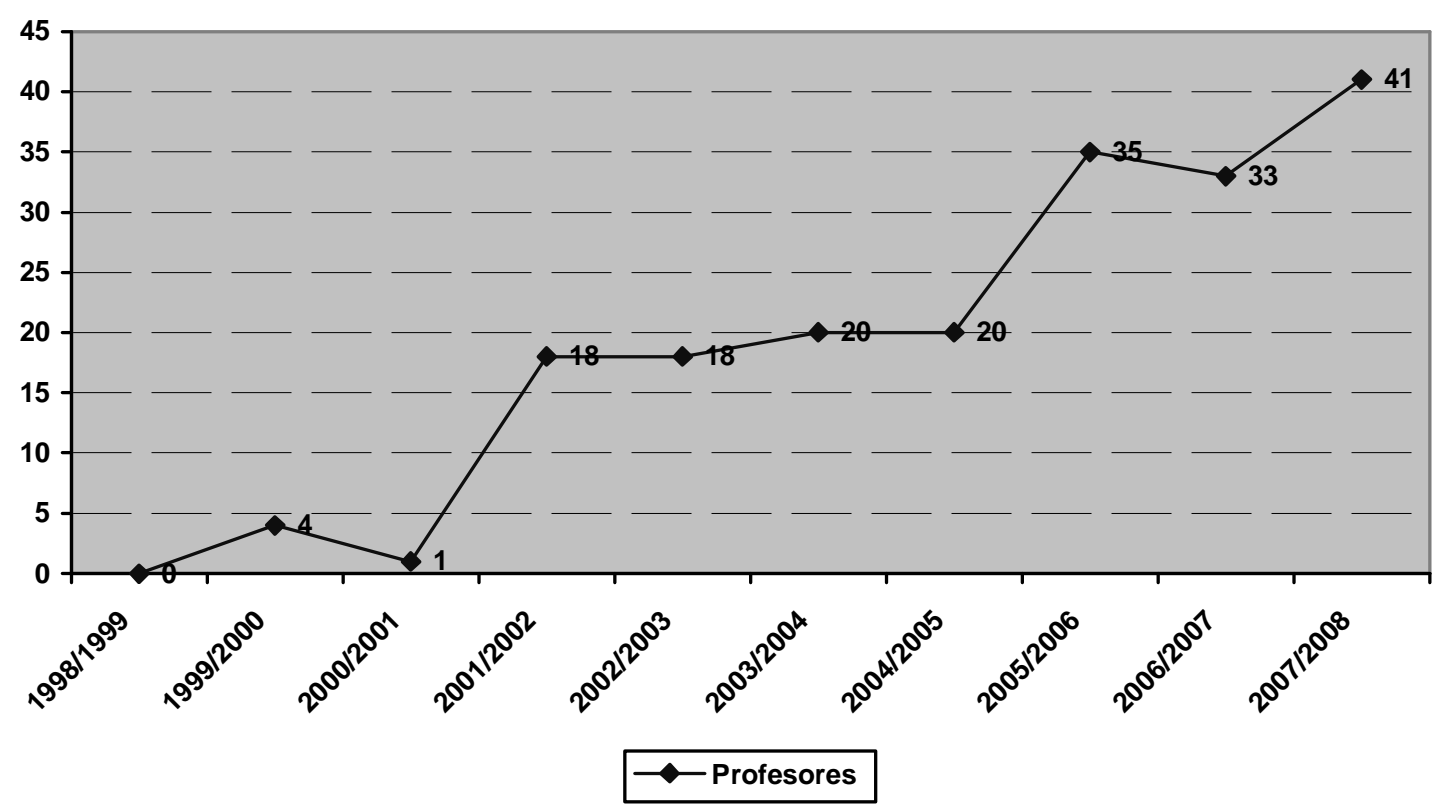

Compte tenu des difficultés pour développer dans la pratique ce qui est juridiquement prévu, difficultés financières, difficultés à trouver un accord pour la désignation des enseignants, les associations musulmanes s'occupent de l'enseignement islamique en dehors de l'école; on voit ainsi fonctionner des écoles coraniques associées aux principales mosquées, l'organisation de cet enseignement n'étant pas subventionnée par l'Etat.

L'on peut donc affirmer que, malgré des évolutions positives en ce sens, l'égal accès à l'éducation religieuse à l'école publique est loin d'être une réalité.

Signalons enfin que parmi les écoles sous contrat en Espagne, escuelas concertadas, la très grande majorité sont catholiques, trois d'entre elles seulement relèvent d'autres confessions, et aucune de l'islam ${ }^{25}$.

En outre, perdure un problème lié à l'évolution des mentalités et de la société dans son ensemble, malgré la revendication officielle du pluralisme et de la place, dans l'histoire et la culture espagnole, de toutes les composantes de sa société. Ceci multiplie les résistances à résoudre les difficultés d'application, surtout si elles se compliquent de problèmes économiques, comme à Ceuta et Melilla. Le président du principal parti de l'opposition, membre du gouvernement jusqu'aux élections de 2004 , avait ainsi déclaré son opposition au fait de « financer l'enseignement de religions qui ne sont pas de notre culture » se référant ici à l'islam. ${ }^{26}$

\section{Conclusion}

La situation actuelle n'est par conséquent, pas encore conforme à la philosophie exprimée dans la Constitution et ses développements normatifs. Pour autant, on ne peut nier que l'Espagne a su trouver des solutions en matière de pluralisme religieux respectueuses à la fois des principes démocratiques et des particularités de son passé, alors qu'elle ne possédait pas de véritable tradition en la matière

Tant dans les conceptions que dans les propos, l'idée de pluralisme joue un rôle fondamental dans le nouveau système mis en place. La dimension verbale et lexicale n'est pas négliger car le recours quasi constant à des mots, des notions clefs, est une des caractéristiques des années postfranquistes, affirmation symbolique, répétée que l'Espagne est devenue une démocratie au travers des thèmes principaux reliés à ces notions.

L'Espagne, plus généralement, a du faire face à deux phénomènes d'intégration : réintégration des siens, avec un succès indéniable, même si des problèmes demeurent ; phénomènes migratoires croissants à partir des années 1980, l'un n'étant pas sans lien avec l'autre. Ainsi, la difficile intégration des immigrés de religion musulmane ne peut s'analyser sans référence à l'histoire de la construction de l'Espagne contre l'islam, et à l'image véhiculée en conséquence. L'état du débat politique

\footnotetext{
${ }^{25}$ Sahuquillo, M.R. 2007. Yahvé y Jehová no van a la escuela, En España sólo hay tres colegios concertados de religiones minoritarias $\varnothing$ La mayoría de estas confesiones defienden que la fe se debe aprender en las iglesias en lugar de en las aulas, El Pais, 01-10-2007

${ }^{26}$ Le Monde, 28 août 2004.
} 
actuel sur ce thème, qui renvoie sans différence notable au débat existant dans les autres démocraties occidentales, peut apparaître comme un signe rassurant sur l'ancrage démocratique et citoyen du système politique espagnol .

De nombreuse mesures sont actuellement mises en oeuvre pour parvenir à une meilleure coïncidence entre droits, pratiques, et, sans doute, évolution des visions sociales. Elles sont très souvent fondées sur l'importance de l'éducation. Le Secrétariat d'Etat à l'Immigration et à l'Emigration du premier gouvernement Zapatero a ainsi mis en place en 2007 un Plan Stratégique, Plano Estratégico de Ciudadaña e Integración afin de donner une nouvelle impulsion aux politiques d'intégration. Il concerne de nombreux domaines, éducation, emploi, logement, services sociaux, santé, jeunesse, lutte contre la discrimination, participation à la vie citoyenne. Il s'agit là d'un cadre d'action, les politiques en matière d'intégration ressortant en Espagne des compétences régionales et locales. Dans le texte ici mentionné, les immigrants sont dénommés « nouveaux citoyens ", par différence avec les citoyens espagnols autochtones. Ce recours à la notion de citoyenneté, et à ses corollaires, égalité, non-discrimination, interculturalité, est significatif de l'importance que l'Etat lui accorde en matière d'intégration. Il implique, entre autres, la reconnaissance de la participation civique, sociale, économique, culturelle et politique des immigrants. L'intégration est aussi définie comme un processus continu d'adaptation mutuelle, avec une référence explicite aux "principes communs gouvernant les politiques d'intégration au sein de l'Union Européenne », qui évoquent "a bidirectional process and dynamic of mutual adjustment for all immigrants and residents of the Member States ». Par conséquent, le nouveau plan s'adresse à la fois aux anciens et aux nouveaux citoyens.

Ces politiques duales sont pensées en termes de vivre-ensemble interculturel, convivencia intercultural, devant permettre une vie dans une société commune basée sur les valeurs de solidarité, de tolérance, de respect, et de reconnaissance des différences religieuses, ethniques et culturelles.

L'accent est enfin mis sur la promotion d'un égal accès aux services publics et privés, au premier rang desquels l'éducation. Les défis en ce domaine sont nombreux, et considérés comme prioritaires. Le texte souligne en particulier la nécessité de considérer l'attention à la diversité comme un principe fondamental à conforter, et souhaite qu'une réponse adéquate soit apportée aux demandes éducatives différentes rendues nécessaires par la diversité des élèves, sans aucune exclusion. Le texte insiste ici, parmi d'autres aspects, sur la régulation de l'enseignement de la religion à l'école et sur l'éducation à la diversité. Certes, le Ministère a ici peu de compétences, le contenu des programmes étant du ressort des Communautés Autonomes. Pour autant, le Ministère prend le soin d'expliquer ses intentions en soulignant qu'un de ses rôles est de favoriser la mise en place de matériaux éducatifs et de promouvoir les meilleures pratiques en matière d'éducation interculturelle. Il a en ce sens créé un centre de Ressources et Données consacrées à la diversité culturelle dans l'éducation $\left(\mathrm{CREADE}^{27}\right)$, site et réseau internet ayant pour but de fournir une aide aux professionnels de l'éducation.

\section{Quelques repères bibliographiques}

\section{La communauté musulmane en Espagne}

Dassetto, F.1996. La construction de l'islam européen. Approche socio-anthropologique. L'Harmattan, Paris

Garretta, J. 2000. Els musulmans de Catalunya. Pagès, Lleida

Moreras, J. 1999. Musulmanes en España. Más allá de la memoria histórica: la viva presencia musulmana en España. CIDOB, Barcelona

Morera, J. 1999. Musulmanes en Barcelona : espacios y dinámicas comunitarias. Cidob Edicions, Barcelona

Motilla, A.(ed.), Los musulmanes en España. Libertad religiosa e identidad cultural. Editorial Trota, Madrid

PLanet, A. 1998. Melilla y Ceuta. Espacios fronteras hispano-marroquíes.UNED-Melilla, Ciudad Autónoma de Melilla, Ciudad Autónoma de Ceuta

Sanchez Nogales, J. L. 2004. El Islam entre nosotros: cristianismo e islam en España. Biblioteca de Autores Cristianos, Madrid

Vicente, Á. (ed.). 2004. Musulmanes en el Aragón del siglo XXI. Instituto de Estudios Islámicos y del Oriente Próximos, Zaragoza

\footnotetext{
${ }^{27} \mathrm{http} / / /$ apliweb.mec.es/creade/index.do
} 
Zapata-Barrero, R. 2006. The Muslim Community and Spanish Tradition: Maurophobia as a Fact, and Impartiality as a Desiratum, In Modood, T., Triandafyllidou, A., and Zapata-Barrero, R. (eds.) Multiculturalism, Muslims and citizenship: a European approach. p.143-161. Routledge, New York

\section{Cadre légal}

Aznar G. F. (ed). 1994. Acuerdos del Estado español con los judíos, musulmanes y protestantes. Publicaciones Universidad Pontificia de Salamanca, Salamanca

Mantecón, J. 2004. Confesiones minoritarias en España : guía de entidades y vademécum normativo. Ministerio de Justicia, Secretaría General Técnica, Madrid

Morera, J. 2001. Limits and contradictions in the legal recognition of Muslims in Spain. In Shadid, W. and Von Koningveld, P. S. (eds). Religious Freedom and the Neutrality of the State : The Position of Islam in the European Union. Peeters, Leiden.

Planet y Contreras, A. 1997. Asociación e Islam : el Acuerdo de cooperación del Estado español con la Comisión Islámica de España y sus repercusiones en Melilla y Ceuta, Estudios sobre el mundo árabe e islámico contemporáneo, vol XVIII : 171-194

\section{Enseignement et multiculturalité}

Aja Fernández, E. and Larios Paterna, M. J. 2003. Inmigración y Educación. Una visión desde la perspectiva jurídica en el Estado Español, interventions aux journées "Inmigración y Educación. La Intervención de la Comunidad Educativa" celebradas en Madrid el 4 y 5 de febrero de 2003, available at http://www.aulaintercultural.org/article.php3?id article=367

Carrasco, S. 1999. Formación de formadores y formadoras en el campo de la interculturalidad. Una experiencia desde la Administracion Educativa de Cataluña. Boletín del Centro de Documentación de la Asociación de Enseñantes con Gitanos, 1617: $47-58$

Council of Europe (2002) "Education for democratic citizenship 2001-2004", Recommendation (2002)12 of the Committee of Ministers to member states on education for democratic citizenship, available at: http://www.seeeducoop.net/education_in/pdf/citizenship2004-oth-enl-t00.pdf Council of the European Union (2004) "Education and citizenship. Report on the broader role of education and its cultural aspects", Brussels: Council of the European Union

Kymlicka, W. 2001. Politics in the vernacular nationalism, multiculturalism, and citizenship. Oxford University Press, Oxford.

Liens :

www.equintanilla.com (articles thématiques)

www.eurel.info (nombreuses références bibliographiques, débats actuels) 